\title{
Reflections on Scattered Light: Monte Carlo Simulations of the Flux and Polarization Curves of $\beta$ Lyrae
}

\author{
Jennifer L. Hoffman \\ Department of Astronomy, University of Wisconsin, 475 N. Charter St., \\ Madison, WI 53706, USA
}

Barbara A. Whitney

Space Science Institute, 1540 30th St., Ste. 23, Boulder, CO 80303, $U S A$

Kenneth H. Nordsieck

Space Astronomy Laboratory, University of Wisconsin, 1150 University Ave., Madison, WI 53706, USA

\begin{abstract}
Polarimetry has proven to be a useful observational tool in the study of Be stars. In recent years, Monte Carlo radiative transfer methods have often been used in modeling polarimetric measurements, since they allow accurate predictions of flux and polarization via numerical simulation of the interaction of light with matter, even in systems with complex configurations. We have extended these two complementary techniques to the investigation of binary Be stars by developing a Monte Carlo code that models the flux and polarization phase curves of a binary-disk system. We here present the latest results matching geometrical model configurations with our own spectropolarimetric observations of the Algol binary $\beta$ Lyrae.
\end{abstract}

\section{The Monte Carlo Code}

Monte Carlo methods rely on random sampling of known small-scale probability distributions to predict large-scale results. Our code emits virtual photons from the stars and disk, then follows each one as it moves through the system. When a photon is inside the disk, the program chooses its interaction path length based on the optical depth of the disk and then decides, based on the disk albedo, whether the photon scatters or is absorbed. At each scattering event, the photon's Stokes parameters are updated; the characteristics of all photons exiting the system are then combined in angle bins. Wavelength may be specified through the choice of albedo values and relative brightnesses of the illuminators.

Our code successfully reproduces the theoretical results of Brown, McLean, \& Emslie (1978) for polarization from an optically thin, symmetric envelope illuminated by point sources. In Figure 1, the dotted ellipses represent BME's predictions for the appropriate inclination angles; they are fitted by eye to our model data points (crosses). 

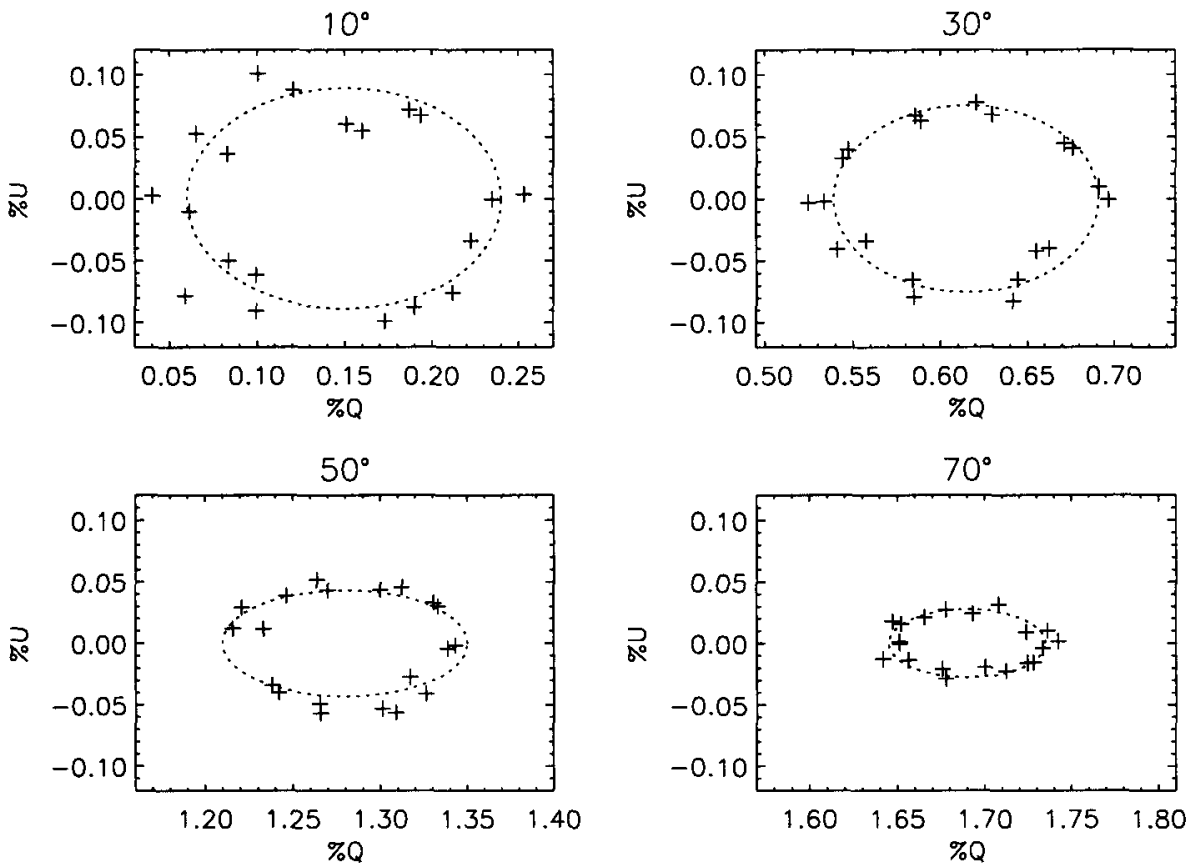

Figure 1. Our model data points overlaid with the theoretical ellipses given by Brown et al. (1978) for various inclination angles.

\section{Model Parameters and Results}

Figure 2 displays the geometrical parameters used in the simulations; these are based on the work of Hubeny \& Plavec (1991).

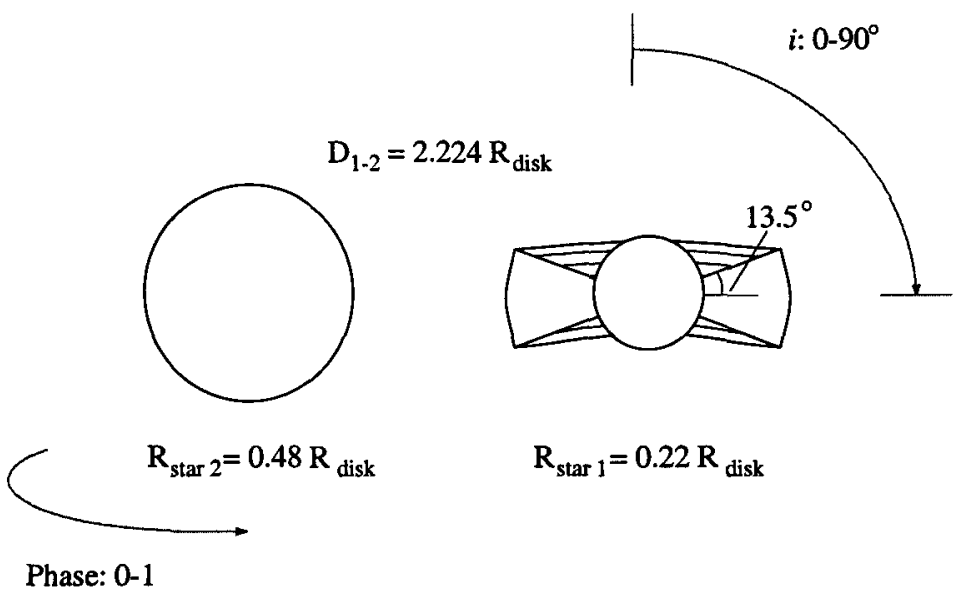

Figure 2. Graphical representation of the geometrical parameters used in our Monte Carlo models. 

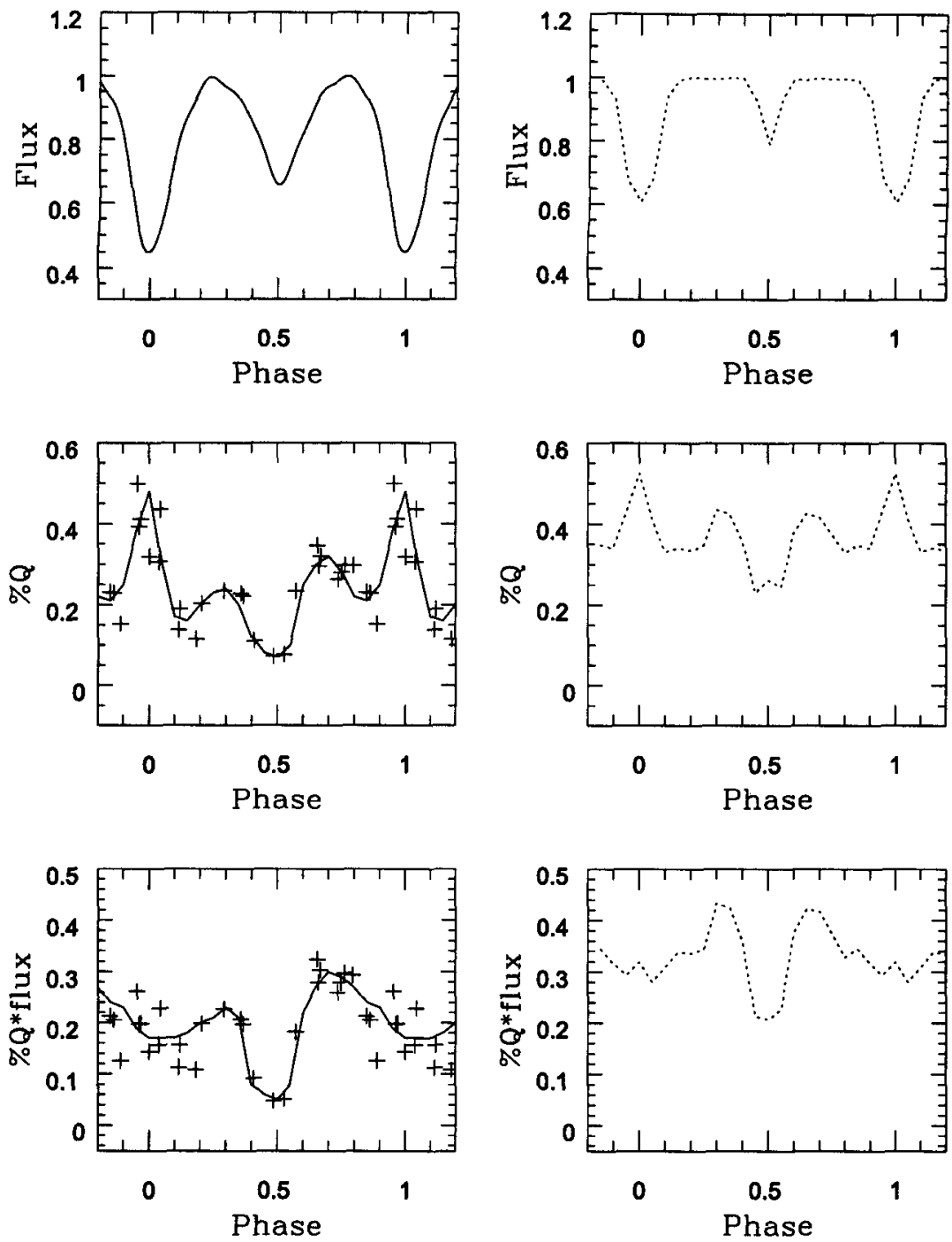

Figure 3. V-band observations of $\beta$ Lyrae (left column) and latest model results (right column).

In the current model, 50 million photons are emitted from three illumination sources, in the ratio star $1:$ star $2:$ disk $=1: 1: 1.33$. The disk's volume emission is proportional to $r^{-2}$, and its optical depth is 5 . A disk albedo of 0.5 simulates the presence of hydrogen bound-free absorption; when photons scatter, they do so via electron scattering. Photons exiting the system are placed into phase angle bins of $18^{\circ}$ width and inclination angle bins of $12^{\circ}$ width; we view the system edge-on, at an inclination of $90^{\circ}$. 
Figure 3 shows V-band observational results in the left column (from Hoffman, Nordsieck, \& Fox 1998), with curves drawn by eye, and results from the above simulation in the right column. The quantities plotted are flux, percent polarization in the direction normal to the disk plane (\%Q), and polarized flux $(\% \mathrm{Q} \times$ flux $)$. The comparison shows that the current model results agree qualitatively with the observed flux and polarization curves of $\beta$ Lyrae, suggesting that the geometrical parameters of the model are reasonable. Quantitative discrepancies indicate that the optical parameters must be adjusted for a more accurate model (see "Future Work" below).

\section{Imaging}

By recording the outgoing direction of each escaping photon, we can create a 3-dimensional model of the system, viewable at any inclination angle and any phase. In order to address the question of the source of the scattered light, we also record where each photon originates; this allows us to make images that are color-coded by photon source. http://www.sal.wisc.edu/ jhoffman (Hoffman's website) features sample images under the heading "Beta Lyrae."

\section{Future Work}

Future modifications to the code will include scattering in a bipolar jet, Roche lobe geometry for the mass-losing star, variation of optical depth within the disk, and consideration of wavelength-dependent effects in hydrogen opacity as well as in the spectra of the illuminators.

\section{References}

Brown, J.C., McLean, I.S., Emslie, A.G. 1978, A\&A 68, 415

Hoffman, J.L., Nordsieck, K.H., Fox, G.K. 1998, AJ 115, 1576

Hubeny, I., Plavec, M.J. 1991, AJ 102, 1156 